\title{
CONDITIONS ON THE CAPTURE-REGION BOUNDARY
}

\author{
YU. A. RYLov \\ Institute for Problems in Mechanics, Academy of Sciences
}

\section{Introduction}

The existence of massless charged particles (electrons and positrons) in the description of the pulsar magnetosphere description leads to the appearance of two phases for each species. In the dynamical phase (DP), the particles move like photons with the speed of light. The static phase (SP), in which the massless particles move with a speed less than that of light, can exist only inside the capture region (C-region). Inside the C-region $(\boldsymbol{E}, \boldsymbol{H})=0$ or $E^{2}<$ $H^{2}$, and the Lorentz force acting upon the particles of $S P$ vanishes. Inside the accelerating region (Aregion), where $(\boldsymbol{E}, \boldsymbol{H}) \neq 0$ or $E^{2} \geq H^{2}$, only the DP can exist.

The pulsar magnetospheric structure is determined by the shape of the A- and C-regions (Rylov 1988). The shape of the capture regions and their boundaries must be determined by dynamical equations. Conditions on the capture-region boundary connect the values of physical quantities in the $\mathrm{A}$ and $\mathrm{C}$-regions. There are a variety of surface $d y-$ namical equations. These conditions are necessary for calculating the magnetospheric structure. Ignorance of these conditions leads to difficulties and inconsistencies (for instance, a singularity on the light cylinder boundary).

In this paper some expressions for discontinuities of the electromagnetic field and its derivatives on the capture-region boundary are obtained and investigated. The capture-region boundaries are classified by types.

\section{Statement of the problem}

In global models of the collisionless pulsar magnetosphere there is a small parameter

$$
\varepsilon=\frac{m c^{2}}{e \Phi}=\frac{m c^{3}}{e \Omega_{0} R H}
$$

where $e, m, \Phi, R, H, \Omega_{0}$ are, respectively, the electron charge, the electron mass, a characteristic electric potential, a characteristic length (radius of the neutron star), the magnetic field, and the angular frequency of the neutron star.

As long as the value of $\varepsilon$ is very small, $\varepsilon=$ $10^{-8}$ to $10^{-11}$, then even the zeroth-order approximation of $\varepsilon$ leads to excellent results. The zeroth- order approximation will be referred to as the massless approximation, because it is associated with a massless charged particle $(m=0 \Rightarrow \varepsilon=0)$. This approximation is good enough at a distance less than $10^{5} R$ to $10^{6} R$. As a rule a charged massless particie has nonzero momentum $p$ and moves with the speed of light. Its velocity $v$ is defined by the electromagnetic field only (Rylov 1989)

$$
\begin{aligned}
v & =v_{\mathrm{S}}=v_{\mathrm{D}}+s v_{\mathrm{L}} \\
s & =\operatorname{sgn}(q)= \pm 1 \\
\boldsymbol{v}_{\mathrm{S}}^{2} & =c^{2}
\end{aligned}
$$

where $q$ is the charge of the particle, $v_{D}$ is the drift component of the particle velocity, and $v_{L}$ is its longitudinal component, which is directed along the magnetic field $\boldsymbol{H}$ in a coordinate system where $\boldsymbol{E} \|$ $\boldsymbol{H}$ (Rylov 1989).

However, there is a very important special case, when $v^{2}<c^{2}$. If

$$
(E, H)=0 \text { or } E^{2}<H^{2}
$$

then the Lorentz force $q\left(\boldsymbol{E}+c^{-1} v \times H\right)$ vanishes. The massless particle can move with a velocity

$$
\boldsymbol{v}=\boldsymbol{v}_{\mathrm{D}}+\alpha \boldsymbol{v}_{\mathrm{L}}, \quad\|\alpha\| \leq 1
$$

where $\alpha$ is arbitrary, but restricted. Its momentum vanishes $p=0$.

The condition of eq.(2) can apply within certain finite regions of the pulsar magnetosphere. These regions will be referred to as capture regions because the particle momentun can be arrested there $(p=0)$. In the capture region two phases of each species can exist at the same time: the dynamical phase (DP) moving with the speed of light [eq.(1)], and the static phase (SP) moving with the velocity given in eq. (3).

The static phase can exist only inside the capture region, whereas the dynamical phase can exist inside both the capture region and the accelerating region where the Lorentz force does not vanish.

The common expression for $v$ has the form

$$
\boldsymbol{v}=\boldsymbol{v}_{\mathrm{D}}+\alpha \boldsymbol{v}_{\mathrm{L}}
$$

where $\alpha \leq 1$ if $(E, H)=0$ and $E^{2}<H^{2}, \alpha=s=$ $\operatorname{sgn}(q)$, if $(E, H) \neq 0$ or $E^{2} \geq H^{2}$. The electrical 
current density $\boldsymbol{j}$ generated by the particles depends on $v$ only, but not on the momentum $p$

$$
j=\rho_{+} v_{1}+\rho_{-} v_{-1} \quad \rho=\rho_{+}+\rho_{-}
$$

where $\rho_{+}$and $\rho_{-}$are the charge densities of the positrons and electrons, respectively.

A self-consistent established flow is described by Maxwell's equations and the continuity equations together with eqs.(4) and (5), where $v_{D}$ and $v_{L}$ are expressed through $\boldsymbol{E}, \boldsymbol{H}$ (Rylov 1989).

On the boundary of the capture region the dynamical variables $\boldsymbol{E}, \boldsymbol{H}, \rho_{+}, \rho_{-}$, and their derivatives have jumps, generally speaking. These jumps can be determined uniquely, because they are corollaries of the system dynamical equations.

There are several types of the jumps which are determined by relations between $v_{\mathrm{Dn}}$ and $v_{\mathrm{Ln}}$ on the capture-region boundary $\Gamma$, where index " $n$ " denotes the normal component $v_{\mathrm{n}}=(\boldsymbol{v}, \boldsymbol{n})$ :

$$
\begin{array}{ll}
\text { I : } & v_{\mathrm{Dn}}^{2}=v_{\mathrm{Ln}}^{2} \\
\text { II }: & v_{\mathrm{Dn}}^{2}<v_{\mathrm{Ln}}^{2} \\
\text { III }: & v_{\mathrm{Dn}}^{2}>v_{\mathrm{Ln}}^{2}
\end{array}
$$

A jump of the normal component $E_{\mathbf{n}}$ of $\boldsymbol{E}$ and non-vanishing surface charge density $\sigma$ appear on the capture-region boundary $\Gamma$ only in case I, when $v_{\mathrm{Dn}}^{2}=v_{\mathrm{Ln}}^{2}$. This is only possible in the case where $\boldsymbol{H}$ is tangent to $\Gamma$. In cases II and III, $\boldsymbol{E}$ and $\boldsymbol{H}$ are continuous on $\Gamma$. Only normal derivatives $\partial \boldsymbol{E} / \partial n$ and $\partial H / \partial n$ can be discontinuous. Mathematical expressions for the jumps can be found in Rylov $(1988,1989,1990)$.

In should be stressed that the size or shape $\Gamma$ of the capture region $\mathrm{V}$ in the pulsar magnetosphere cannot be given. They depend on the dynamical equations of which the capture boundary conditions are a surface form. The shape of $\Gamma$ cannot be determined without using the conditions on the captureregion boundary.

In particular, in many first generation pulsar models (the models of early seventies (Goldreich and Julian 1969, Julian 1973, Michel 1973, Scharlemann and Wagoner 1973) the whole magnetosphere is supposed to consist of the capture region only. This leads to singularities on the light cylinder and to other inconsistencies.

In second generation pulsar models, where the capture-region boundary conditions are taken into account, these inconsistencies are removed.

\section{The case of charge separation}

In this case there are particles of only one species (electrons or positrons) at any point. Let us say that the boundary $\Gamma$ is unstable with respect to species $s(s=1$ for positrons and $s=-1$ for electrons) if there is an outflow of $s$ through $\Gamma$, $\left(v_{s}, n\right)>0 . \Gamma$ is stable with respect to the species $s$, if there is no outflow of $s$ through $\Gamma\left(v_{s}, n\right) \leq 0$.

In case II, when $v_{\mathrm{L}}$ dominates $v_{\mathrm{D}},\left(v_{\mathrm{Ln}}^{2}>v_{\mathrm{Dn}}^{2}\right)$, $\Gamma$ can be stable with respect to positrons and unstable with respect to electrons (case $\mathrm{II}_{+}$). Conversely, $\Gamma$ can be stable for electrons and unstable for positrons (case II_-).

In case III, when $v_{\mathrm{D}}$ dominates $\boldsymbol{v}_{\mathrm{L}},\left(v_{\mathrm{Dn}}^{2}>\right.$ $\left.v_{\mathrm{Ln}}^{2}\right), \Gamma$ can either be stable for both electrons and positrons (case $\mathrm{III}_{+}$) or unstable for both (case III $_{0}$ ).

In the coordinate systems of cases II and III, $K_{\text {II }}$ and $K_{\mathrm{III}}$, where, respectively, $v_{\mathrm{Ln}}=0$, one has

$$
\begin{array}{ll}
\mathrm{II}_{+}: & v_{\text {Dn }}=0, \quad v_{\text {Ln }}<0 ; \\
\mathrm{II}_{-}: & v_{\text {Dn }}=0, \quad v_{\text {Ln }}>0 ; \\
\mathrm{III}_{ \pm}: & v_{\text {Ln }}=0, \quad v_{\text {Dn }}<0 ; \\
\mathrm{III}_{0}: & v_{\text {Ln }}=0, \quad v_{\text {Dn }}>0 .
\end{array}
$$

The types of capture-region boundaries in axisymmetric charge-separated pulsar magnetospheres with net total charge are shown schematically in figure 1.

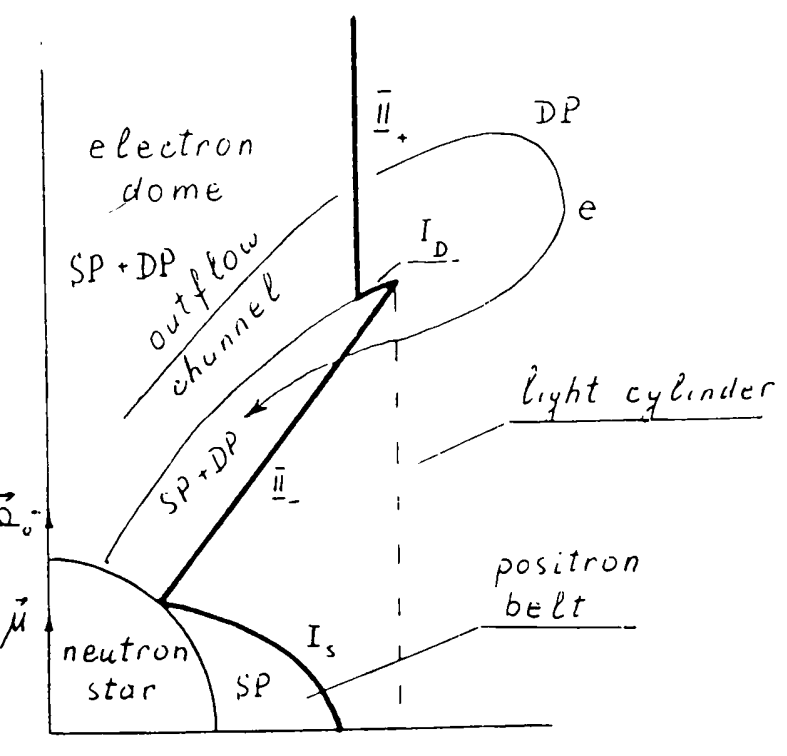

Figure 1

In case I following types of capture-region boundaries exist:

$$
\begin{array}{ll}
I_{\mathrm{D}+}: & v_{\mathrm{Ln}}=-v_{\mathrm{Dn}} \neq 0 ; \\
I_{\mathrm{D}-}: & v_{\mathrm{Ln}}=v_{\mathrm{Dn}} \neq 0 ; \\
I_{\mathrm{S}}: & v_{\mathrm{Ln}}=v_{\mathrm{Dn}}=0 .
\end{array}
$$

Case $I_{\mathrm{D}+}$ corresponds to a positron, and case $I_{\mathrm{D}-}$ to an electron surface charge. Case $I_{\mathrm{S}}$ corresponds to asurface charge constructed from SP.

Case $I_{S}$, when a non-vanishing surface density of captured particles (SP) arises in empty space, seems to be impossible. Nevertheless, very large charge density (simulating the surface charge) can arise in the pulsar magnetosphere, as figures 1 and 4 of Krause-Polstorff and Michel (1985), where the magnetosphere was calculated numerically for the case of large positive total charge. 